\title{
An Update on Device Cleaning Technologies
}

\author{
Gerald McDonnell ${ }^{1}$, Peter A. Burke ${ }^{2}$ \\ ${ }^{1}$ STERIS Ltd, Basingstoke, United Kingdom \\ ${ }^{2}$ STERIS Corporation, Ohio, USA.
}

doi: 10.3396/ijic.V5i2.020.09

The reprocessing of reusable medical devices (and indeed any patient-contact surface) is performed in a series of steps to essentially include cleaning, disinfection and/or sterilization. ${ }^{1}$ The emphasis of infection prevention practices has been traditionally on the antimicrobial aspects of these steps; however, in recent years greater emphasis is being placed on the efficacy, safety, and reproducibility of cleaning. This has been driven by adverse patient reports, scientific investigations, and new guidelines/standards. In clinical practice, the old adage of 'if a surface is not clean it can not be disinfected/sterilized' is the primary reason for this focus, but not the only one. For example, one of the major causes of device damage is when patient soils are allowed to dry and remain on surfaces for extended times. Of particular note are adverse patient reaction reports due to contact with devices that have contained low levels of various soils; examples include pyrogenic reactions (due to the presence of bacterial toxins such as endotoxins), TASS (Toxic Anterior Segment Syndrome), ${ }^{2}$ and dramatically transmission of fatal disease (associated with CJD). ${ }^{3}$

Cleaning is defined as the removal of contamination from an item to the extent necessary for its further processing and its intended subsequent use. The science of cleaning has focused on defining the level and types of soil components (such as proteins, lipids, etc) before and after cleaning, defining the analytical endpoints and test methods for a 'clean' surface, the design of artificial test soils and cleaning indicators for testing purposes. Defining an endpoint for cleaning is not as straight forward as it seems. The simple requirement is for visual cleanliness, but this would not apply to areas of the device that can not be directly observed (e.g., in lumens), is subjective and variable. A consolidation of published literature certainly has a consensus that the detection of residual protein is the most reliable method, with typical levels for a cleaning endpoint being in the range of $\leq 6.4 \mu \mathrm{g} / \mathrm{cm}^{2}$. However, this level would apply if indeed this is shown not to interfere with subsequent disinfection and sterilization. For example, if the disinfectant is aldehyde-based this level of protein may become fixed onto the device over multiple cycles, potentially leading to future adverse events. Similarly, much lower levels of prion proteins on surfaces have been shown to be infectious (but it is important to note that there is much yet to be learned in consideration of disease transmission in these cases). ${ }^{4}$ Despite these considerations, the defined level of protein seems as a useful and practical level for determining a minimum cleaning endpoint. This level (and significantly lower) can be routinely determined under laboratory conditions using a variety of analytical methods and, at a minimum can be routinely confirmed in clinical practice. ${ }^{5} \mathrm{~A}$ further 
consideration is the design of representative test soils for laboratory and clinical test purposes, as well as the design of cleaning indicators for the routine testing/ verification of cleaning efficacy. ${ }^{5}$ Examples of cleaning test kits, indicators and artificial test soils are shown in Figure 1. It should be noted that close attention should be given to the variety of 'cleaning' indicators and test methods becoming available; for example, some indicators are only designed to detect microbial contamination (e.g., ATP-based methods) that may not correlate to levels of proteins or other soils on device surfaces and may therefore provide misleading results.
Similar to sterilization, the assurance of a consistent and reproducible process is important to cleaning. New standards and guidelines (e.g., ISO 15883 series on washer-disinfectors) highlight the importance of a defined process, to include the washer-disinfector design, associated racks/baskets, cycle definition, cleaning chemistry and water quality. These requirements have driven new innovations, to include new cleaning chemistries and washer-disinfector design. Cleaning chemistries are now specifically designed for medical device applications. Advances with these products include safe transport formulations to ensure devices are not allowed to dry on transport
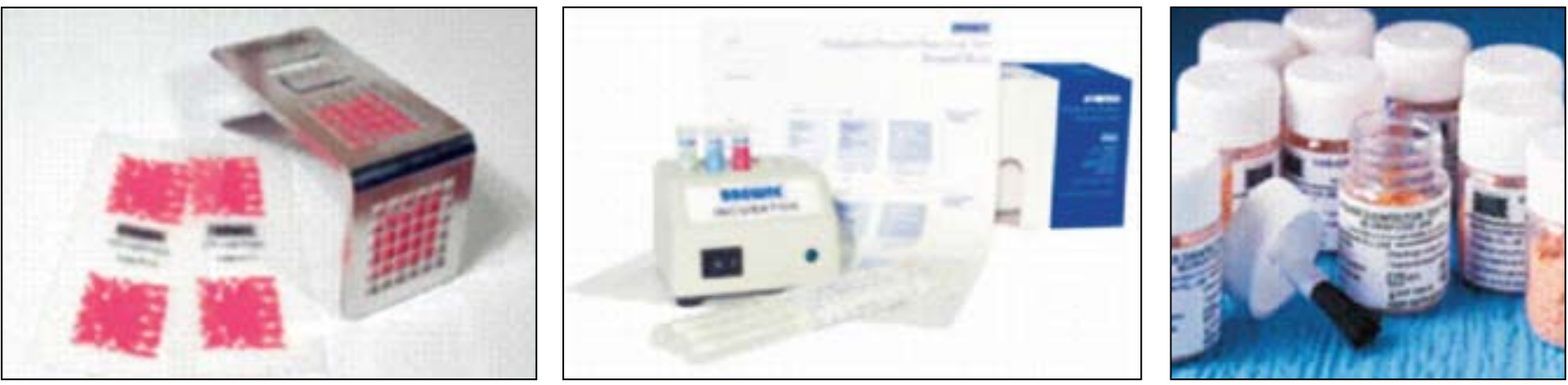

Figure 1: A cleaning indicator (left), cleaning endpoint test kit (based on a swab test using ninhydrin; middle) and artificial test soil (right) for use in the monitoring of cleaning processes.

to a reprocessing area, optimal cleaning performance (balanced with tolerance of varying water quality and device compatibility), proven efficacy to reduce the risks of prion contamination ${ }^{4}$ and environmental considerations.

There is also a trend away from using manual cleaning to more reliable washer-disinfectors. Washer-disinfector designs have improved considerably relative to the new standards and with many new innovations, to include water/energy consumption optimization, short cycle times, cleaning-disinfection cycle definition/ validation, water filtration, process assurance etc.

Cleaning will continue to be important in device reprocessing but should now be considered as being applied a part of a consistent and validated process. It not only can play a role to ensure reliable disinfection and sterilization but can improve patient safety, be environmentally friendly and allow for the handling of a wider range of complex instruments and accessories.

\section{References}

1. ISO 17664:2004. Sterilization of medical devices. Information to be provided by the manufacturer for the processing of resterilizable medical devices.

2. Werner L, Sher JH, Taylor JR, et al. Outbreak of toxic anterior segment syndrome and possible association with ointment in the anterior chamber following cataract surgery. I Cataract Refract Surg 2006; 32: 227-235.

3. Bernoulli C, Siegfried J, Baumgartner G, et al. Danger of accidental person-to-person transmission of Creutzfeldt-Jakob disease by surgery. Lancet 1977; 1(8009): 478-479.

4. McDonnell G. Prion disease transmission: can we apply standard precautions to prevent or reduce risks? Journal of Perioperative Practices 2008; 18(7): 298-304.

5. McDonnell G. Soils and soil detection methodology: Current and Emerging Technology. IDSC Journal 2006; 10(4): 16-20. 